Original Research Article

\title{
Molecular Docking Studies of Spirostans as MAPK14 (P38a) Inhibitors and Their Potential Use against Cancer
}

\section{Guiee Niza Lopez-Castillo ${ }^{1}$}

Victorino Alatriste 2

Jesus Sandoval-Ramírez ${ }^{3}$

Felix Luna 2

Alan Carrasco-Carballo ${ }^{*}$ (6)

1Laboratorio de Elucidación y Síntesis en Química Orgánica, Facultad de Ciencias Químicas, Benemérita Universidad Autónoma de Puebla, Puebla, Puebla, Mexico

2Laboratorio de Neuroendocrinología, Departamento de Farmacia, Facultad de Ciencias Químicas, Benemérita Universidad Autónoma de Puebla, Puebla, Puebla, Mexico

${ }^{3}$ Laboratorio de Síntesis y Modificación de Productos Naturales, Facultad de Ciencias Químicas, Benemérita Universidad Autónoma de Puebla, Puebla, Puebla, Mexico

*email: alan.carrascoc@correo.buap.mx

Keywords:

MAPK14 inhibition

Molecular docking

Spirostans

\begin{abstract}
Spirostans (SPs) are chemical products widely distributed in the plant kingdom; currently, they are studied by their medical applications. Cancer has a high incidence in humans; it reaches second place worldwide deaths. In molecular biology, it has been accepted that Mitogen-Activated Protein p38alpha Kinase (MAPK14 (p38a) is implicated in the regulation of cancer. This study aimed to identify SPs as potential MAPK14 (p38a) inhibitors. From a set of 133 modified SPs, SwissTargetPrediction platform, and molecular docking, it was obtained that 129 chemical structures had molecular interaction with the MAPK14 (p38a). From those molecules, 123 were bound to a specific inhibition site of MAPK14 (p38a), and 6 of the structures resulted in inhibitors similarly to minocycline and dasatinib. One SP had binding couple energy (BCE, $\mathrm{kcal} / \mathrm{mol}$ ) as that of fostamatinib. In addition, 115 modified SPs had better BCE than the minocycline but not as that using fostamatinib. The key amino acids (aa) for the protein kinase MAPK14 (p38a) inhibition were Arg 70, Asp 168, Lys 53, His 148 , and Ile 145, at a different interaction level. The BCE was enhanced when the $\mathrm{H}$ atom was substituted in $\mathrm{C}-2, \mathrm{C}-11$, and $\mathrm{C}-17 \mathrm{SPs}$ positions. Similarly, the aOH group at C-5 and C-6 upgraded BCE. Stereochemistry and substitution at C-3, C-12, and C-25 did not present significant differences (Kruskal-Wallis test, $p<0.05$ ). From all this ensemble of results, it is foreseeable that the SPs can be an option for MAPK14 (p38a) inhibition, a key modulator in cancer processes.
\end{abstract}

Received: November 14th, 2021

Accepted: December 20th, 2021

Published: December 30th, 2021

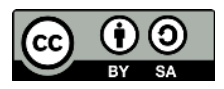

(C) 2021 Guiee Niza Lopez-Castillo, Victorino Alatriste, Jesus Sandoval-Ramírez, Felix Luna, Alan CarrascoCarballo. Published by Institute for Research and Community Services Universitas Muhammadiyah Palangkaraya. This is an Open Access article under the CC-BY-SA License (http://creativecommons.org/licenses/by-sa/4.0/). DOI: https://doi.org/10.33084/jmd.v1i2.2904

\section{INTRODUCTION}

Spirostans (SPs) are obtained from the acidic hydrolysis of the corresponding saponins, which are abundant in the plant kingdom ${ }^{1}$. Some SPs (Figure 1) have shown biological activity in many human pathologies such as polycystic ovary syndrome, metabolic syndrome, rheumatoid arthritis, breast, cervical, and prostate cancer, among others ${ }^{2-9}$. The SPs bioactivity has been attributed to their chemical structure; in this order, specifically to substituents in various stereocenters of the steroidal nucleus and the ring F. The scientific literature has also reported the important role of SPs anticancer molecules ${ }^{10-13}$.

Based on characteristics described previously, the SPs are interesting molecules and can be studied against cancer. In cancer, cells are exposed to constant changes by the local environment, changes in metabolism, and general, in cell physiology. Recent data have proposed that the Mitogen-Activated Protein Kinase family (MAPKs) is involved in the cancer process that affects many tissues involved in human diseases. The MAPK family, specifically MAPK ( $\mathrm{p} 38)$ proteins that include MAPK14 (p38a), MAP11 (p38ß), MAPK12 (p38Y and 
MAPK13 (p38ס) was reported in alterations in the growth signals, protection, and apoptotic cell death, de novo angiogenesis, and metastasis ${ }^{14}$.

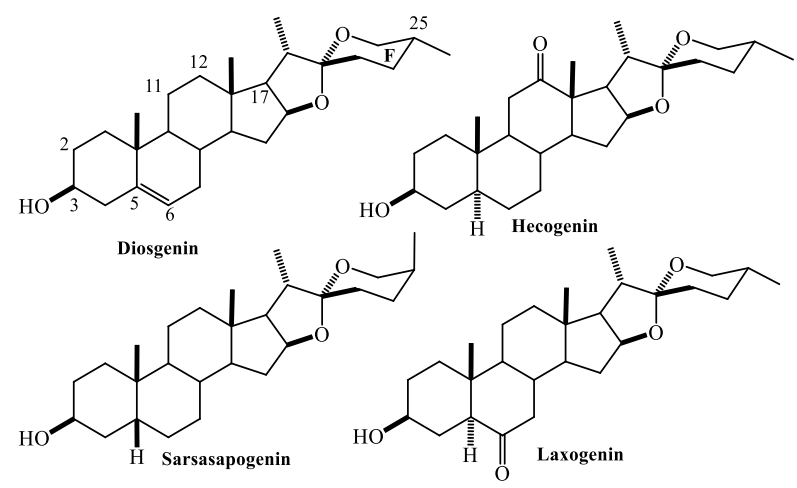

Figure 1. Some biological active SPs.

This manuscript focused on MAPK14 (p38a) as SPs molecular target. MAPK14 has been reported as a suppressor of tumorigenesis ${ }^{14}$ because it is expressed in most cell types and is involved in pancreatic, renal cell carcinoma 15,16 , breast cancer, ovarian serous adenocarcinoma, rhabdomyosarcoma-derived cell, among other cancers ${ }^{17}$. Our in silico data shows a potential role of SPs as inhibitors for MAPK1417-19. So, the study of SPs as inhibitors for MAPK $14^{20}$ (Figure 2) can open a new way to understand their action mechanism and test more accurately new compounds as a new treatment against cancer.

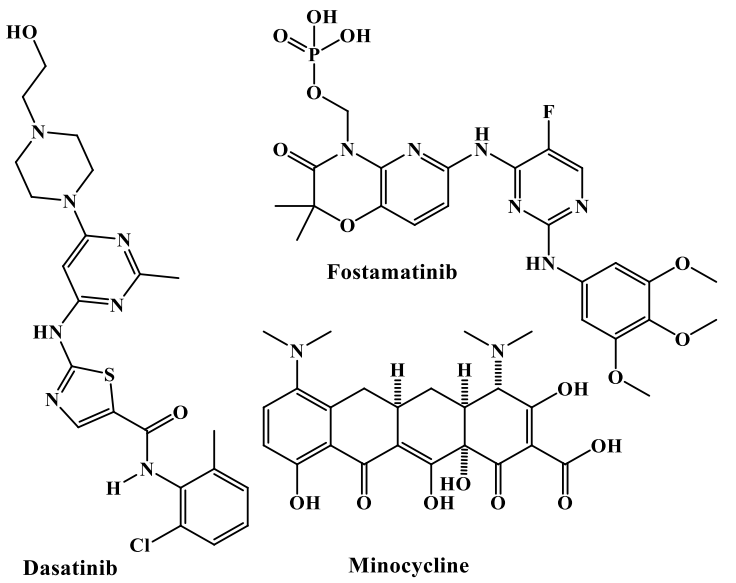

Figure 2. Commercial MAPK14 inhibitors of DrugBank Online (https://go.drugbank.com/).

\section{METHOD}

\section{Hardware and Software}

It was used PC with Intel® CoreTM i9-10900 RAM 64.00 GB @ $2.80 \mathrm{GHz}, 64$-bit operating system at Windows $10 . \quad$ Also, the software SwissTargetPrediction

(STP; www.swisstargetprediction.ch/ $)^{21}, \quad$ ChemDraw
Professional 17.1, Chem3D 17.1, Discovery Studio ${ }^{\circledR}$ (www.3ds.com/), AutoDockTools $®$ (http://autodock.scripps.edu/resources/adt)22, and AutoDock Vina ${ }^{23}$.

\section{Ligands}

The reference drugs were set of DrugBank Online (https://go.drugbank.com/); minocycline (DB01017), dasatinib (DB01254), fostamatinib (DB120102) ${ }^{24}$, and SPs were designed using ChemDraw 17.1.

\section{Receptors}

The receptor used as the molecular target was MAPK14 (p38a) bound to SR348, downloaded from protein data bank (https://www.rcsb.org/) as PDB code 6ZWP25.

\section{Docking protocol}

The optimized energy and geometry for the ligand structures were calculated using $\mathrm{MM}^{26}$ and PM7 (http://openmopac.net/) in Chem3D 17.1 and MOPAC. The protein MAPK14 (p38a) was prepared by adding polar hydrogens and optimized in AutoDock Tools ${ }^{22}$. Redocking with SR348 obtained at RMSD $0.87 \AA$ A. The molecular docking was calculated with AutoDock Vina ${ }^{23}$.

\section{Assessment}

The biological potential for SPs was evaluated through STP. Then, the molecular coupling studies of SPs were analyzed compared to drugs reported in the DrugBank database. First, in a punctual way towards specific sites of MAPK14 (p38a) and interactional study energy, later, the binding coupling energy (BCE) values were obtained. The results were compared with Kruskal-Wallis ${ }^{27}$, and $\mathrm{p}<0.05$ was considered statistically different. The describers were defined with a significant effect on the BCE value (Figure 3).
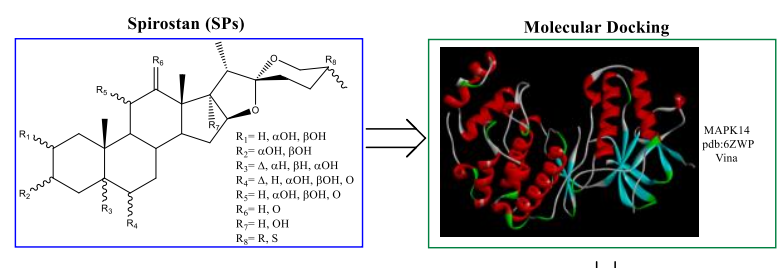

$\Downarrow$

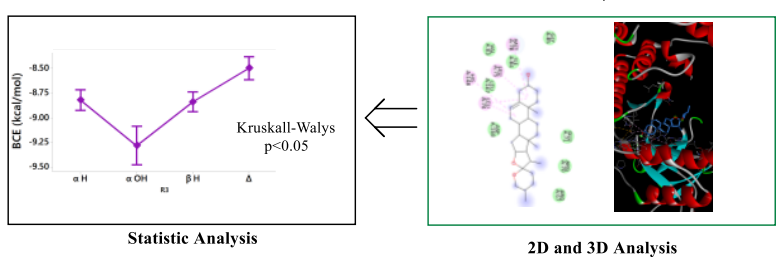

Figure 3. Methodology used for the study of SPs. 


\section{RESULTS AND DISCUSSION}

For commercial MAPK14 (p38a) inhibitors, minocycline, dasatinib, and fostamatinib, the BCE obtained were $-8.0,-8.2$, and $-9.8 \mathrm{kcal} / \mathrm{mol}$, respectively (Figure 4). These molecules were joined to the MAPK14 (p38a) specific site by interactions with the amino acid residues Lys A:53, Arg A:67, Leu A:74, Met A:78, Ile A:146, His A:148, Leu A: 167 and Asp A:168; in each case, it was different interaction types on the MAPK14's inhibition site in comparison to reference compounds. The BCE value was related to the interaction type and the amino acid residue it interacts with. Asp A:168 interacted by a conventional hydrogen bond with minocycline and dasatinib. Lys A:53 had the same type of bond with dasatinib and fostamatinib, and the $\triangle B C E \sim 1.0 \mathrm{kcal} / \mathrm{mol}$ between minocycline and fostamatinib was related to amino acids residues Arg A:149 and Arg A:67 with unfavorable acceptor-acceptor and bump interactions, Asp A:168 and Glu A:71 was by sulfur-X interactions. In our studied SPs group ( $\mathrm{n}=133) ; 26 \%$ are naturals, $24 \%$ synthetic and $50 \%$ are not reported so far. Two SPs presented the same BCE to minocycline. One is better but smaller than the dasatinib, six with similar BCE values, 123 with better BCE than dasatinib but worse than fostamatinib, and just one with the same energy as the last one (Figure 5). The SPs present better potential inhibitory effect for MAPK14 (p38a) than dasatinib and minocycline but not than fostamatinib. Besides, $98 \%$ of the SPs were in the MAPK14 (p38a) specific site, between the central loop and the central ahelices of the A chain (Figure 6), except in three SPs
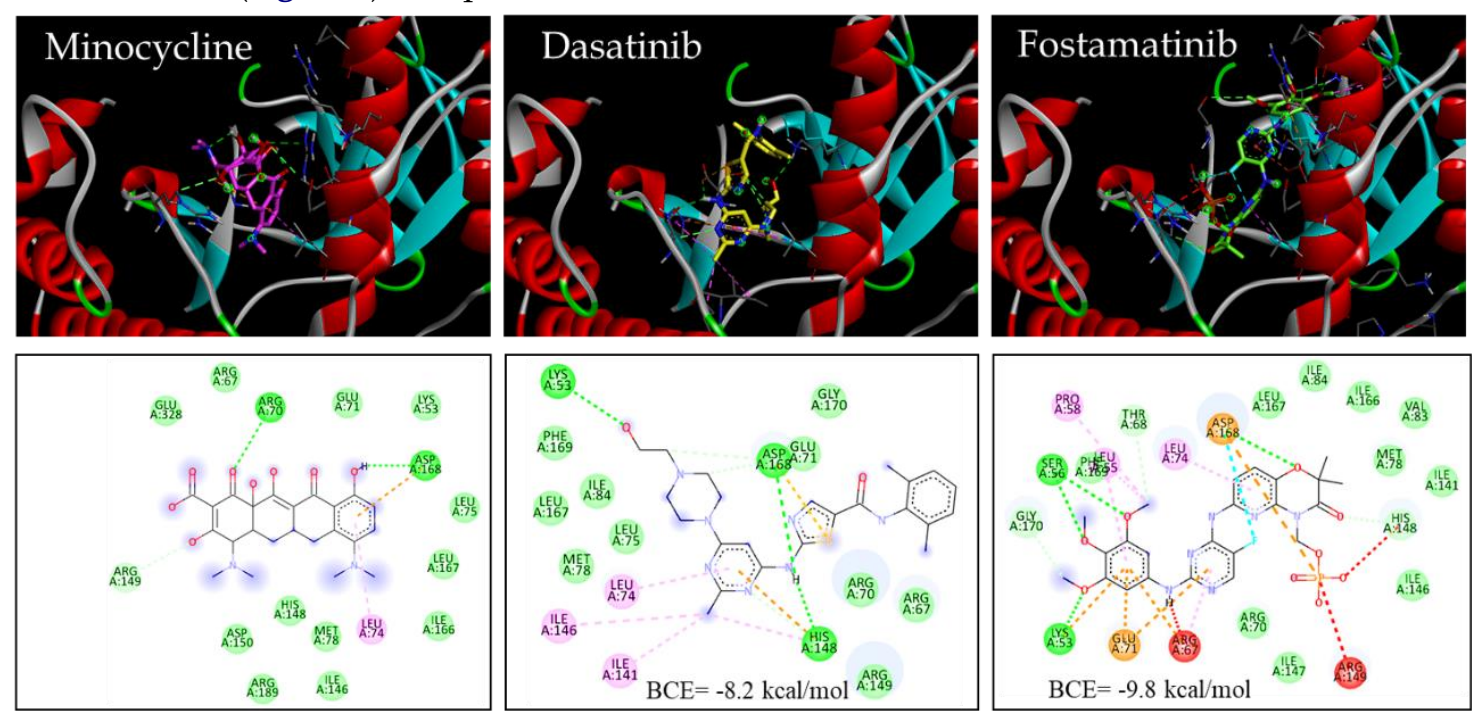

Interactions $\square$ Conventional Hydrogen Bond $\square$ Vander Waals $\square$ Pi-Alky $\square$ Alkyl

Figure 4. 3D and 2D interactions of reference drugs in MAPK14 (p38a). with the worst $\mathrm{BCE}$ value. The arrangement of these compounds was found in the $\beta$-stacked sheets supported by the spiroketal moiety towards the nearby loop. In 3D models, the interaction site was amphipathic, the internal zone was hydrophobic, and the external zone hydrophilic. The steroids were located on the part of the steroidal nucleus in the hydrophobic zone and the oxygens of the spiroketal in the hydrophilic region, except for the 6-oxygenated derivatives compounds which have more significant molecular interaction on the hydrophilic site.

The BCE depends on the interactions of each one of the compounds present with the target protein. Figure 7 shows the selected SPs with the descriptors, demonstrating the effect caused on the coupling force by a functional group with specific stereochemistry. In C-2, the coupling improvement SP-1, which has hydrogen in this carbon, due to an acetyl group in equatorial position (SP-17), promotes hydrogen bridge formation with the protein. In contrast, for SP-24, whose structural difference is the stereochemistry of C-3 in the axial position, the coupling force decreased even more than SP-1 and can be explained by the interactions Van der Waals. For the stereochemistry of C-25, better interaction was observed between amino acid residues and SP-114 and SP-123 with equatorial methyl with p-alkyl type interaction. Unlike SP-90, whose methyl is axial, which only has p-s interactions, these SPs presented better coupling than SP-1 and SP24, which has a double bond at C-5 described before, comparing the hydrogen interactions in this same position was observed similarity by their nature. 


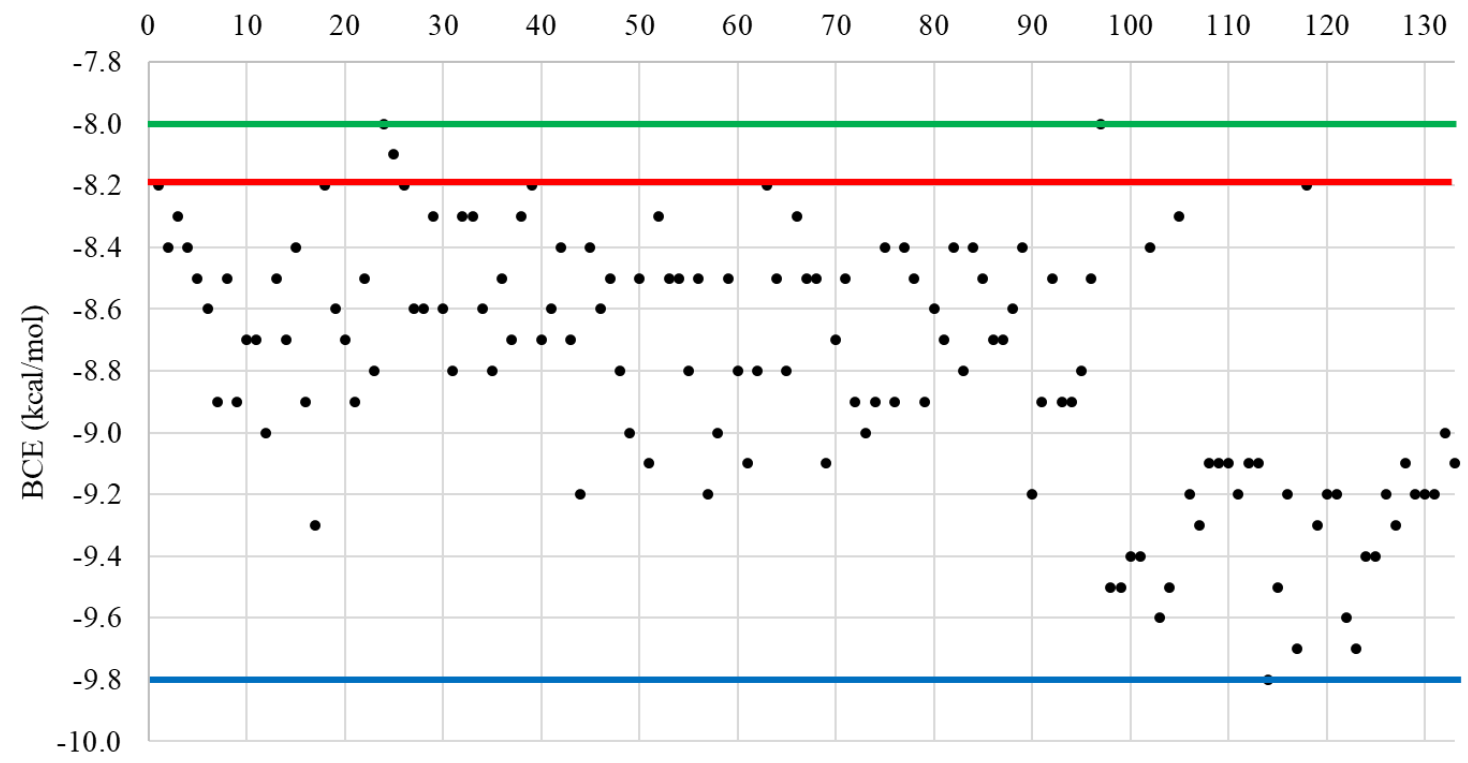

Figure 5. Comparative BCE (kcal/mol) to MAPK14 (p38a), for SPs and drug references minocycline (green line), dasatinib (red line), and fostamatinib (blue line).
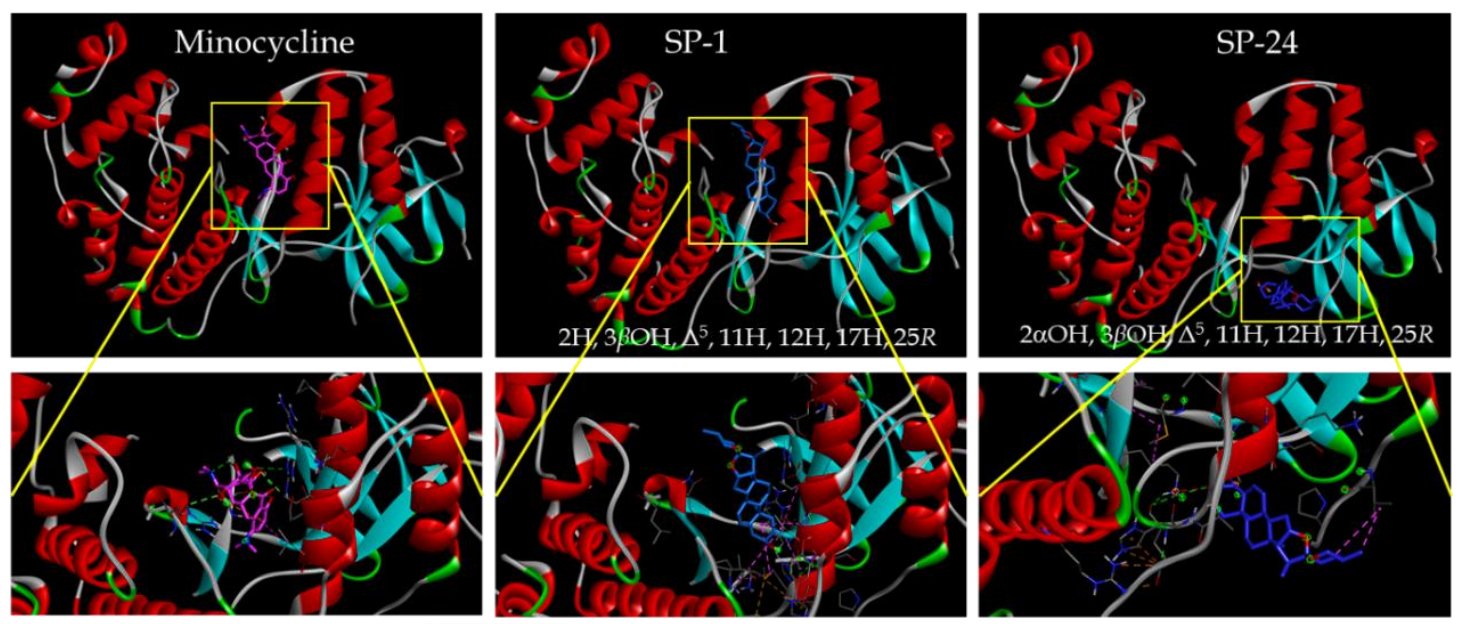

Interactions $\square$ Conventional Hydrogen Bond $\square$ Van der Waals $\square$ Pi-Alky $\square$ Alkyl $\square$ Unfavorable Acceptor-Acceptor $\square$ Unfavorable Bump $\square$ Pi-Sigma $\square$ Sulfur-X

Figure 6. 3D model and interactions in specific site of MAPK14 (p38a).

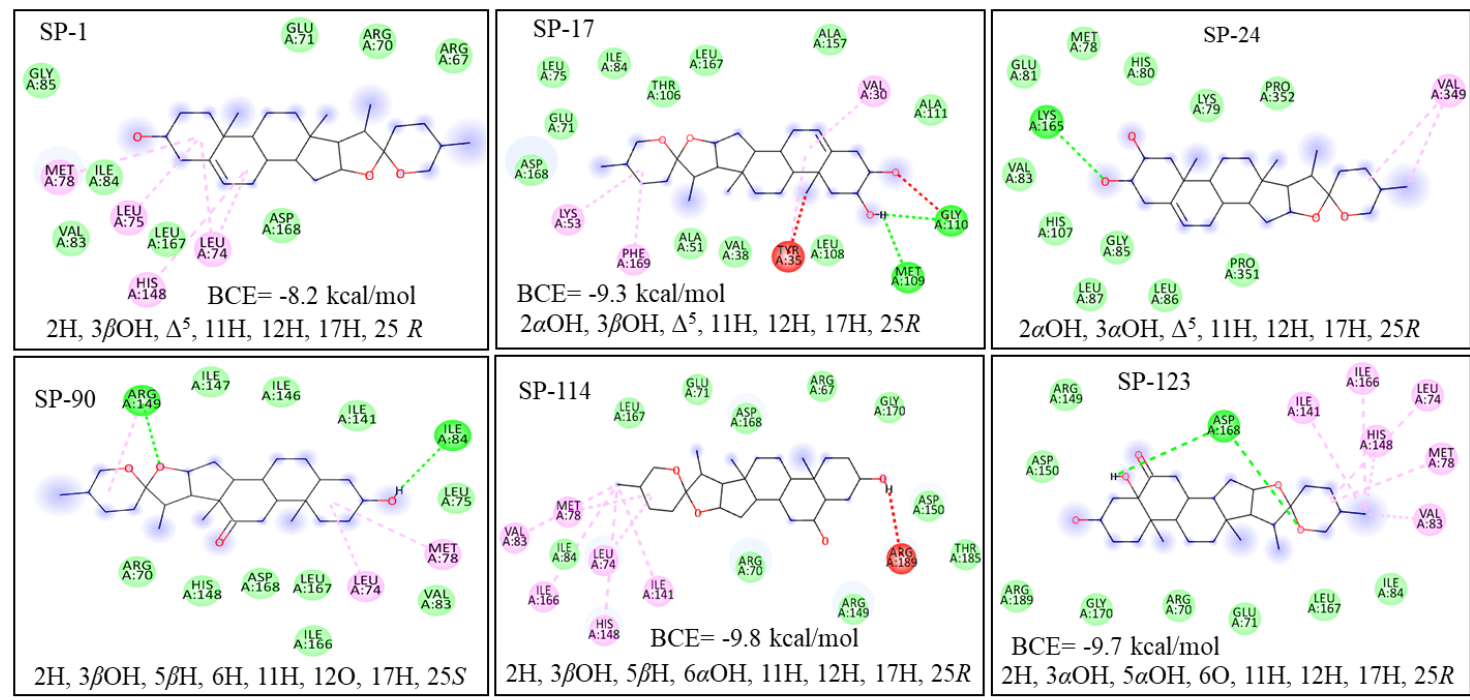

Interactions $\square$ Conventional Hydrogen Bond $\square$ Vander Waals $\square$ Pi-Alkyl $\square$ Alkyl $\square$ Unfavorable Acceptor-Acceptor $\square$ Unfavorable Bump $\square$ Pi-Sigma

Figure 7. 2D diagram interactional of SPs in the specific site of MAPK14 (p38a). 
For the $\mathrm{C}-12$, the change in hybridization and polarity in the $\mathrm{C}$ ring rearranged the interaction with the MAPK14 (p38a) specific site. For SP-114 and SP-123, van der Waals and p-alkyl interactions stand out. For the first one, it is important to highlight that having equatorial hydrogen at C-5 and axial hydroxyl at C-6 was better for an unfavorable interaction with hydroxyl at C-328,29. SS-123 with an axial hydroxyl group at C-5 promoted the hydrogen bridge-type interaction with the protein target, leaving the interaction with the carbonyl at C-6 and the hydroxyl at C-3.

The grade of interactional similarity was found by analyzing the residues of the MAPK14 (p38a) with the commercial inhibitors and SPs interact (Table I). However, SP-24 presented the same BCE as minocycline; SP-24 only interacts with Met A:78 because the SP is outside the active site. For dasatinib, whose BCE was $-8.2 \mathrm{kcal} / \mathrm{mol}$, its energetic counterpart was SP-1. However, SPs had stronger hydrogen bridge and sulfur interactions than the control inhibitor.

The interaction with amino acid residues presented similarity close to $80 \%$, characterizing a compound with similar BCE to the commercial inhibitor drug without sulfur and nitrogen atoms in its structure. The same benefit can be observed in SP-17 and SP-90, whose BCE value was between dasatinib and fostamatinib energy. Besides, SP-90 presented a similar pattern in the interaction residues, whereas SP17 presented interactional differences with the residues due to its low similarity, only in $33 \%$. The presence of hydrogen bridges was shown with high coupling energy. For the fostamatinib energetic to SP114 , it is observed that the interaction residues have a close $80 \%$ match with van der Waals and p-alkyl type interactions. However, in this case, the unfavorable interaction with Arg A:189 stands out, which benefits the molecular coupling despite not being on the residues list of the commercial inhibitor. In the case of the SP-123 interactions $(-9.7 \mathrm{kcal} / \mathrm{mol})$, energetically was close to the commercial inhibitor fostamatinib, whose interactional match was $70 \%$, this high value can be attributed to hydrogen bridge with Asp A:168, which is on the residues list of fostamatinib.

With this analysis, it was found that Lys A: 53, Arg A: 67, Arg A: 70, Leu A: 74, Met A: 78, Ile A: 46, His A: 148, Leu A: 167, and Asp A: 168 are key amino acids for the inhibition of MAPK14 (p38a), a target important for the cancer treatment. These data, which also corroborated with the SP, could highlight the Asp A:168 residue whose interaction was observed with all the structures analyzed in the previous stage; Met A:78, which is only absent in one of the SPs. However, it is the only main residue observed with the SP-24 outside the active site, and its interaction remains due to the location of the amino acid in the lower zone of the a-helix.

Detailed studies permitted us to know the interaction between SPs and MAPK14 (p38a). Additionally, statistical analysis allows us to analyze the effect of each descriptor ${ }^{22}$. Non-parametric Kruskal-Wallis statistics were carried out for data that were not normally distributed, which was summarized in Table II. There were no changes in the stereogenic centers at C-3, C-12, and C-25. In C-25 (Figure 8), the side chain is exposed towards the area between chains $\mathrm{A}$ and $\mathrm{B}$, which explains the null effect; the same case is for $\mathrm{R} 2$. In the case of $\mathrm{C}-12$, the $\mathrm{C}$ ring is placed in this space between the chains, generating a null effect on the $\mathrm{BCE}^{30}$.

For descriptors with significant differences to the exposed region, the steroids compounds are coupled over the side, which explains why the modifications on it a more remarkable influence have, as the case of C-6 (Figure 9) when this was oxygenated ${ }^{31}$. The position was exchanged, leaving the hydrophobic part of compounds exposed to the hydrophobic and hydrophilic sites, which considerably increased in the BCE. The same effect occurred in the C-5 hydroxyl group; it increased the hydrophilic region in rings $\mathrm{A}$ and B. In the case of substitution by hydrogen, there was no difference in its stereochemistry because it preserves the no polar environment of the region, but when it did present a double bond, the BCE decayed due to the hybridization change decreasing the contact area of receptor ${ }^{32}$. In C-17, it did present better energy when it has hydrogen as a substituent because it changes the polar environment in the D and $\mathrm{E}$ ring region, becoming polar when it is in the hydrophobic region $^{33}$. At C-11, the carbonyl group or the hydrogen atom showed better BCE due to the hydrophobic region or only the hydrogen bond donor, but not the acceptor, as with hydroxyl substitutions in the molecular structure. 
Table I. Molecular docking between MAPK14 amino acid and reference drugs or SPs

\begin{tabular}{|c|c|c|c|c|}
\hline Inhibitors & $\begin{array}{c}\text { BCE } \\
\text { (kcal/mol) } \\
\text { to APK14 }\end{array}$ & van der Waals and alkyl interactions & $\begin{array}{l}\text { Hydrogen } \\
\text { bond } \\
\text { interaction }\end{array}$ & $\begin{array}{l}\text { Unfavorable acceptor- } \\
\text { acceptor, sulfur-X, and } \\
\text { halogen bond interactions }\end{array}$ \\
\hline Minocycline & -8.0 & $\begin{array}{l}\text { Lys A:53; Arg A:67; Glu A:71; Leu A:74; Leu A:75; Met A:78; Ile } \\
\text { A:146; His A:148; Arg A:149; Asp A:150; Ile A:166; Leu A:167; Arg } \\
\text { A:189; Glu A:328 }\end{array}$ & $\begin{array}{l}\text { Arg A:70; } \\
\text { Asp A:168 }\end{array}$ & Asp A:168 \\
\hline Dasatinib & -8.2 & $\begin{array}{l}\text { Arg A:67; Arg A:70; Glu A:71; Leu A:74; Leu A:75; Met A:78; Ile A:84; } \\
\text { Ile A:141; Ile A:146; Arg A:149; Leu A:167; Phe A:169; Gly A:170 }\end{array}$ & $\begin{array}{l}\text { Lys A:53; } \\
\text { His A:148; } \\
\text { Asp A:168 }\end{array}$ & His A:148; Asp A:168 \\
\hline Fostamatinib & -9.8 & $\begin{array}{l}\text { Leu A:55; Pro A:58; Thr A:68; Arg A:70; Leu A:74; Met A:78; Val } \\
\text { A:83; Ile A:84; Ile A:141; Ile A:146; Ile A:147; His A:148; Ile A:166; Leu } \\
\text { A:167; Phe A:169; Gly A:170 }\end{array}$ & $\begin{array}{l}\text { Lys A:53; } \\
\text { Ser A:56; } \\
\text { Asp A:168 }\end{array}$ & $\begin{array}{l}\text { Lys A:53; Arg A:67; His } \\
\text { A:148; Asp A:168 }\end{array}$ \\
\hline SP-1 & -8.2 & $\begin{array}{l}\text { Arg A:67; Arg A:70; Glu A:71; Leu A:74; Leu A:75; Met A:78; Val } \\
\text { A:83; Ile A:84; Gly A:85; His A:148; Leu A:167; Asp A:168 }\end{array}$ & - & - \\
\hline SP-17 & -9.3 & $\begin{array}{l}\text { Val A:30; Tyr A:35; Val A:38; Ala A:51; Lys A:53; Glu A:71; Leu A:75; } \\
\text { Ile A:84; Thr A:106; Leu A:108; Ala A:111; Ala A:157; Leu A:167; Asp } \\
\text { A:168; Phe A:169 }\end{array}$ & $\begin{array}{l}\text { Met A:109; } \\
\text { Gly A:110 }\end{array}$ & Tyr A:35; Gly A:110 \\
\hline SP-24 & -8.0 & $\begin{array}{l}\text { Met A:78; Lys A:79; His A:80; Glu A:81; Val A:83; Gly A:85; Leu A:86; } \\
\text { Leu A:87; His A:107; Val A:349; Pro A:351; Pro A:352 }\end{array}$ & Lys A:165 & - \\
\hline SP-90 & -9.2 & $\begin{array}{l}\text { Arg A:70; Leu A:74; Leu A:75; Met A:78; Val A:83; lle A:141; Ile } \\
\text { A:146; Ile A:147; His A:148; Arg A:149; Ile A:166; Leu A:167; Asp } \\
\text { A:168 }\end{array}$ & $\begin{array}{l}\text { lle A:84; } \\
\text { Arg A:149 }\end{array}$ & - \\
\hline SP-114 & -9.8 & $\begin{array}{l}\text { Arg A:67; Arg A:70; Glu A:71; Leu A:74; Met A:78; Val A:83; lle A:84; } \\
\text { Ie A:141; His A:148; Arg A:149; Asp A:150; Пle A:166; Leu A:167; Asp } \\
\text { A:168; Gly A:170; The A:185 }\end{array}$ & - & Arg A:189 \\
\hline SP-123 & -9.7 & $\begin{array}{l}\text { Arg A:70; Glu A:71; Leu A:74; Met A:78; Val A:83; Ile A:84; Ile A:141; } \\
\text { His A:148; Arg A:149; Asp A:150; Ile A:166; Leu A:167; Gly A:170; } \\
\text { Arg A:189 }\end{array}$ & Asp A:168 & - \\
\hline
\end{tabular}

Bold letters indicate key amino acid residues

Table II. Statistical results of SPs stereogenic centers on BCE. Kruskal-Wallis test, $\mathrm{p}<0.05$

\begin{tabular}{|c|c|c|c|c|c|c|c|}
\hline $\mathbf{D}$ & $\mathbf{R}$ & Statistical difference & & & Best BCE & & \\
\hline $\mathrm{C} 2$ & 1 & Yes & & $\mathrm{H}$ & & $\mathrm{aOH}, \beta \mathrm{OH}$ & \\
\hline C3 & 2 & No & & & Independent of stereochemistry at C-3 & & \\
\hline C5 & 3 & Yes & $\mathrm{aOH}$ & & $\alpha \mathrm{H}, \beta \mathrm{H}$ & & $\Delta$ \\
\hline $\mathrm{C} 6$ & 4 & Yes & $\mathrm{O}, \mathrm{aOH}$ & & $\beta \mathrm{OH}$ & & $\Delta$ \\
\hline $\mathrm{C} 11$ & 5 & Yes & & $\mathrm{H}, \mathrm{O}$ & & $\mathrm{aOH}, \beta \mathrm{OH}$ & \\
\hline $\mathrm{C} 12$ & 6 & No & & & Independent of substitution at C-3 & & \\
\hline $\mathrm{C} 17$ & 7 & Yes & & $\mathrm{H}$ & & $\mathrm{aOH}$ & \\
\hline $\mathrm{C} 25$ & 8 & No & & & Independent of stereochemistry at C-25 & & \\
\hline
\end{tabular}

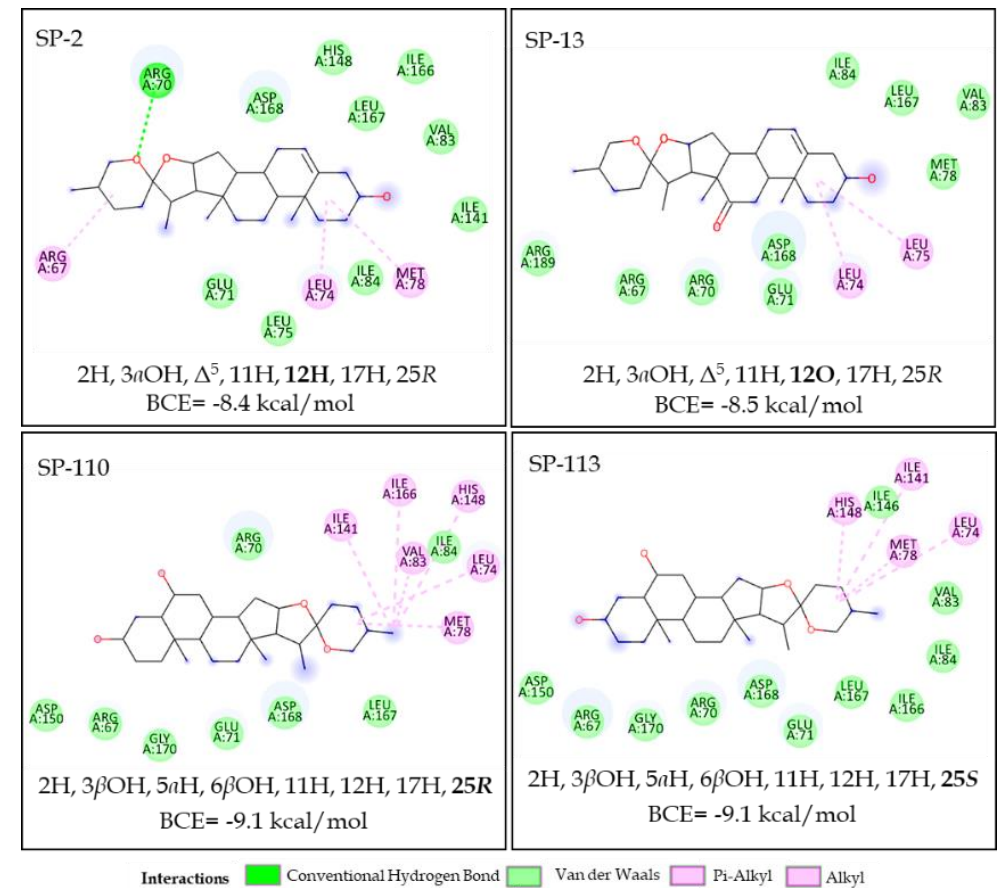

Figure 8. SPs without statistical difference in C-12 (A) and C-25 (B). Kruskal-Wallis test, p >0.05. 


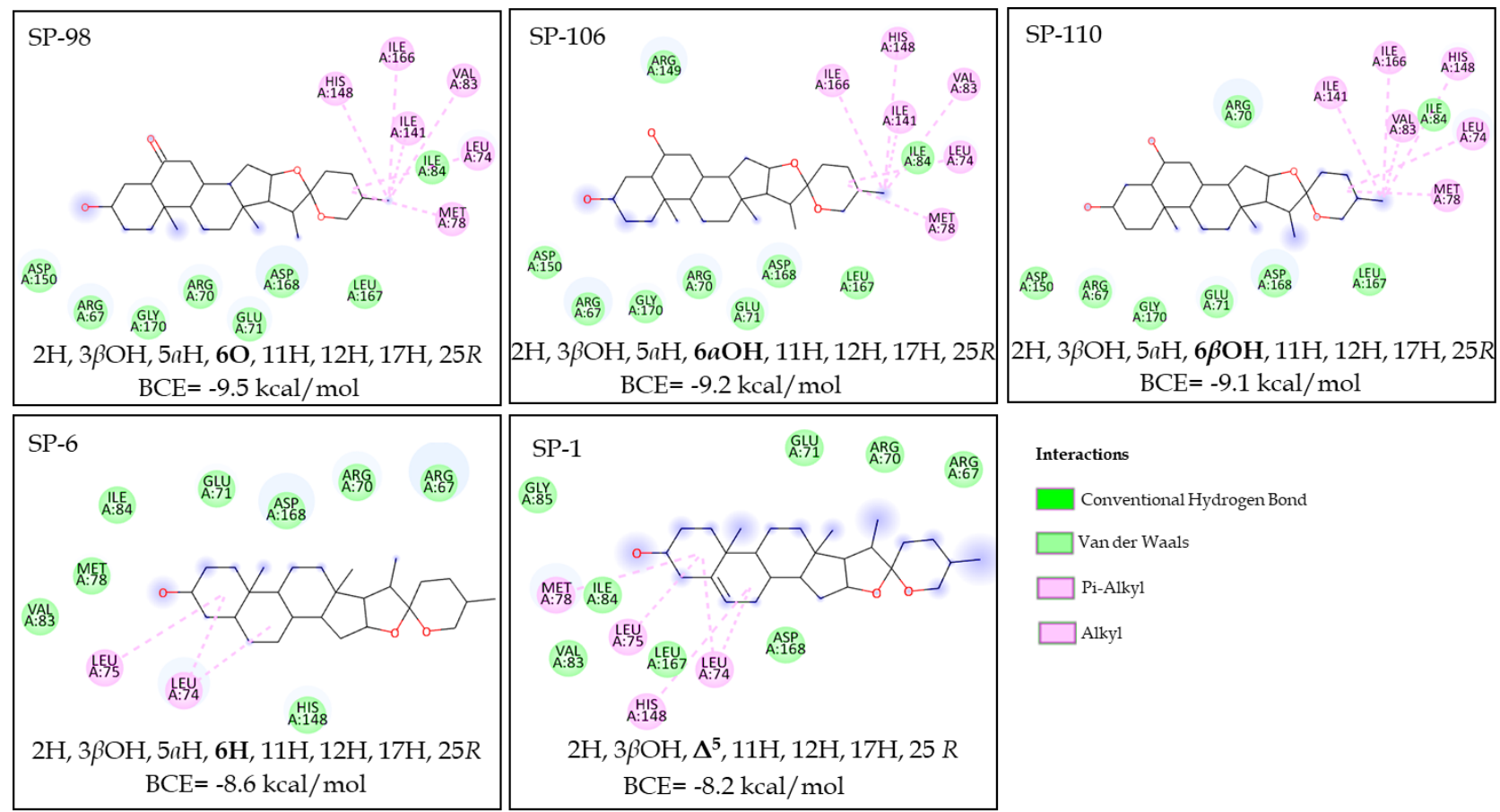

Figure 9. SPs with statistical difference in C-6 at MAPK14. Kruskal-Wallis test $\mathrm{p}<0.05$.

\section{CONCLUSION}

Molecular docking studies predict a favorable interaction between MAPK14 (p38a) and SPs, with $\mathrm{BCE}$ in between the range of two widely used commercial inhibitors. Only two of the 133 SPs did not bind at the specific MAPK14 (p38a) protein site. This result can be attributed to the most repetitive interaction van der Waals type between the key amino acids: Lys 53, Arg 67, Arg 70, Leu 74, Met 78, Ile 46, His 148, Leu 167, and Asp 168, also the carbonyl, hydroxyl, hydrogen, and methyl substituents which are in specific positions in the study compounds. Hydrogen bond interaction between Asp 168 and the hydroxyl group of SP-123 presented the best coupling with the MAPK14 (p38a) protein. From the analysis of interaction energies, it was very favorable the presence of hydrogen in C-2, C-17, and C-11. In addition, the last one also improved being a carbonyl group. The same effect was observed in C-6 and C-5; the presence of a hydroxyl group favored the interaction energy. These results support that SPs 17, 114 y 123 were the better MAPK14 (p38a) inhibitors and suggest that they can be potential therapeutic agents in cancer treatment.

\section{CONFLICTS OF INTEREST}

The authors have no conflicts of interest to declare that are relevant to the content of this article.

\section{FUNDING}

Consejo Nacional de Ciencia y Tecnología (CONACYT) for grant 826153-16989 (SNI-3 assistant program) and PN331.

\section{DATA AVAILABILITY}

All data are available from the authors. The software tools and version for docking analyses and specifics modules used. AutoDock Vina and SwissTargetPrediction can be used with a free license.

\section{ACKNOWLEDGMENTS}

Guiee Niza Lopez-Castillo acknowledge CONACYT for grant 826153-16989 (SNI-3 assistant program) and PN331.

\section{AUTHORS' CONTRIBUTIONS}

Guiee Niza Lopez-Castillo: methodology, resources, software. Victorino Alatriste: formal analysis, investigation, resources, writing. Jesus SandovalRamírez: formal analysis, writing - review \& editing. Felix Luna: formal analysis, supervision, validation, visualization, writing - review \& editing. Alan Carrasco-Carballo: conceptualization, data curation, software, formal analysis, investigation, writing. 


\section{REFERENCES}

1. Jastrzebska I. Chemistry of steroidal sapogenins new advances in a classical field. Curr Org Chem. 2012;16(3):353-72. doi:10.2174/138527212799499895

2. Tang ZZ, Zhang YM, Zheng T, Huang TT, Ma TF, Liu YW. Sarsasapogenin alleviates diabetic nephropathy through suppression of chronic inflammation by down-regulating PAR-1: In vivo and in vitro study. Phytomedicine. 2020;78:153314. doi:10.1016/j.phymed.2020.153314

3. Liagre B, Vergne-Salle P, Leger DY, Beneytout JL. Inhibition of human rheumatoid arthritis synovial cell survival by hecogenin and tigogenin is associated with increased apoptosis, p38 mitogenactivated protein kinase activity and upregulation of cyclooxygenase-2. Int J Mol Med. 2007;20(4):45160. doi:10.3892/ijmm.20.4.451

4. Wang W, Zhang Y, Yao G, Wang W, Shang X, Zhang $Y$, et al. Synthesis of new sarsasapogenin derivatives with antiproliferative and apoptotic effects in MCF-7 cells. Steroids. 2018;131:23-31. doi:10.1016/j.steroids.2018.01.001

5. Hilario-Martínez JC, Huerta A, Amaro-López JC, Alatriste V, De los Santos MG, Martínez I, et al. Stereoselective synthesis of (26R)-26hydroxydiosgenin and its effect on the regulation of rat ovarian function. Bioorg Chem. 2021;115:105189. doi:10.1016/j.bioorg.2021.105189

6. Sánchez-Sánchez L, Escobar ML, SandovalRamírez J, López-Muñoz H, Fernández-Herrera MA, Hernández-Vázquez JMV, et al. Apoptotic and autophagic cell death induced by glucolaxogenin in cervical cancer cells. Apoptosis. 2015;20(12):1623-35. doi:10.1007/s10495-015-11816

7. Hernández-Vázquez JMV, López-Muñoz $\mathrm{H}$, Escobar-Sánchez ML, Flores-Guzmán F, WeissSteider B, Hilario-Martínez JC, et al. Apoptotic, necrotic, and antiproliferative activity of diosgenin and diosgenin glycosides on cervical cancer cells. Eur J Pharmacol. 2020;871:172942. doi:10.1016/j.ejphar.2020.172942

8. Escobar-Sánchez ML, Sánchez-Sánchez L, Sandoval-Ramírez J. Steroidal Saponins and Cell Death in Cancer, Cell Death - Autophagy, Apoptosis and Necrosis. IntechOpen. 2015. p. 33151. doi:10.5772/61438
9. Carrasco-Carballo A, Hernández-Linares MG, Cárdenas-García M, Sandoval-Ramírez J. Synthesis and biological in vitro evaluation of the effect of hydroxyimino steroidal derivatives on breast cancer cells. Steroids. 2021;166:108787. doi:10.1016/j.steroids.2020.108787

10. Corbière C, Liagre B, Bianchi A, Bordji K, Dauça M, Netter P, et al. Different contribution of apoptosis to the antiproliferative effects of diosgenin and other plant steroids, hecogenin and tigogenin, on human 1547 osteosarcoma cells. Int J Oncol. 2003;22(4):899-905. doi:10.3892/ijo.22.4.899

11. Acharya D, Mitaine-Offer AC, Kaushik N, Miyamoto T, Paululat T, Mirjolet JF, et al. Cytotoxic spirostane-type saponins from the roots of Chlorophytum borivilianum. J Nat Prod. 2009;72(1):177-81. doi:10.1021/np800559z

12. Wang W, Meng H. Cytotoxic, anti-inflammatory and hemostatic spirostane-steroidal saponins from the ethanol extract of the roots of Bletilla striata. Fitoterapia. 2015;101:12-8. doi:10.1016/j.fitote.2014.11.005

13. Xu XH, Li T, Fong CMV, Chen X, Chen XJ, Wang $\mathrm{YT}$, et al. Saponins from Chinese medicines as anticancer agents. Molecules. 2016;21(10):1326. doi:10.3390/molecules21101326

14. Cuenda A, Rousseau S. p38 MAP-Kinases pathway regulation, function and role in human diseases. Biochim Biophys Acta. 2007;1773(8):135875. doi:10.1016/j.bbamcr.2007.03.010

15. Liu J, Yu X, Yu H, Liu B, Zhang Z, Kong C, et al. Knockdown of MAPK14 inhibits the proliferation and migration of clear cell renal cell carcinoma by downregulating the expression of CDC25B. $\begin{array}{lll}\text { Cancer } & \text { Med. 2020;9(3):1183-95. }\end{array}$ doi:10.1002/cam4.2795

16. Yang L, Sun X, Ye Y, Lu Y, Zuo J, Liu W, et al. p38a Mitogen-Activated Protein Kinase Is a Druggable Target in Pancreatic Adenocarcinoma. Front Oncol. 2019;9:1294. doi:10.3389/fonc.2019.01294

17. Mesquita FP, Moreira-Nunes CA, da Silva EL, Lima LB, Daniel JP, Zuerker WJ, et al. MAPK14 (p38a) inhibition effects against metastatic gastric cancer cells: A potential biomarker and pharmacological target. Toxicol In Vitro. 2020;66:104839. doi:10.1016/j.tiv.2020.104839 
18. Dashti S, Taherian-Esfahani Z, Kholghi-Oskooei V, Noroozi R, Arsang-Jang S, Ghafouri-Fard S, et al. In silico identification of MAPK14-related lncRNAs and assessment of their expression in breast cancer samples. Sci Rep. 2020;10:8316. doi:10.1038/s41598-020-65421-2

19. Ding QY, Zhang Y, Ma L, Chen YG, Wu JH, Zhang $\mathrm{HF}$, et al. Inhibiting MAPK14 showed antiprolactinoma effect. BMC Endocr Disord. 2020;20:138. doi:10.1186/s12902-020-00619-z

20. Gu L, Tao X, Xu Y, Han X, Qi Y, Xu L, et al. Dioscin alleviates BDL- and DMN-induced hepatic fibrosis via Sirt1/Nrf2-mediated inhibition of p38 MAPK pathway. Toxicol Appl Pharmacol. 2016;292:19-29. doi:10.1016/j.taap.2015.12.024

21. Daina A, Michielin O, Zoete V. SwissTargetPrediction: updated data and new features for efficient prediction of protein targets of small molecules. Nucleic Acids Res. 2019;47(W1):W357-W364. doi:10.1093/nar/gkz382

22. Sanner MF, Jolla L. News and Views. J Mol Graph Model. 1999;17(1):55-84. doi:10.1016/S10933263(99)99999-0

23. Trott O, Olson AJ. Software News and Update AutoDock Vina: Improving the Speed and Accuracy of Docking with a New Scoring Function, Efficient Optimization, and Multithreading. J Comput Chem. 2010;31:455-61. doi:10.1002/jcc.21334

24. Wishart DS, Feunang YD, Guo AC, Lo EJ, Marcu A, Grant JR, et al. DrugBank 5.0: A major update to the DrugBank database for 2018. Nucleic Acids Res. 2018;46(D1):D1074-D1082. doi:10.1093/nar/gkx1037

25. Röhm S, Schröder M, Dwyer JE, Widdowson CS, Chaikuad A, Berger BT, et al. Selective targeting of the aC and DFG-out pocket in p38 MAPK. Eur J
Med Chem. 2020;208:112721. doi:10.1016/j.ejmech.2020.112721

26. Allinger NL. Conformational analysis. 130. MM2. A hydrocarbon force field utilizing V1 and V2 torsional terms. J Am Chem Soc. 1977;99(25):812734. doi:10.1021/ja00467a001

27. Kruskal WH, Wallis WA. Use of Ranks in OneCriterion Variance Analysis. J Am Stat Assoc. 1952;47(260):583-621. doi:10.2307/2280779

28. Lee KH, Schnupf U, Sumpter BG, Irle S. Performance of Density-Functional Tight-Binding in Comparison to $\mathrm{Ab}$ Initio and First-Principles Methods for Isomer Geometries and Energies of Glucose Epimers in Vacuo and Solution. ACS Omega. 2018;3(12):16899-915. doi:10.1021/acsomega.8b02213

29. Qiu L, Lin J, Liu Q, Wang S, Lv G, Li K, et al. The Role of the Hydroxyl Group in Propofol-Protein Target Recognition: Insights from ONIOMStudies. J Phys Chem B. 2017;121(24):5883-96. doi:10.1021/acs.jpcb.7b02079

30. Torres PHM, Sodero ACR, Jofily P, Silva Jr FP. Key Topics in Molecular Docking for Drug Design. Int J Mol Sci. 2019;20(18):4574. doi:10.3390/ijms20184574

31. Miller WL, Auchus RJ. The molecular biology, biochemistry, and physiology of human steroidogenesis and its disorders. Endocr Rev. 2011;32(1):81-151. doi:10.1210/er.2010-0013

32. Erbas-Cakmak S, Leigh DA, McTernan CT, Nussbaumer AL. Artificial Molecular Machines. Chem Rev. 2015;115(18):10081-206. doi:10.1021/acs.chemrev.5b00146

33. Sarkar A, Kellogg GE. Hydrophobicity--shake flasks, protein folding and drug discovery. Curr Top Med Chem. 2010;10(1):67-83. doi:10.2174/156802610790232233 